\title{
Médiévales
}

Langues, Textes, Histoire

66 | printemps 2014

Harmonie Disharmonie

\section{Harmonie et disharmonie}

\section{Christopher Lucken et Ludivine Jaquiery}

\section{OpenEdition}

Journals

Édition électronique

URL : https://journals.openedition.org/medievales/7181

DOI : $10.4000 /$ medievales. 7181

ISSN : 1777-5892

\section{Éditeur}

Presses universitaires de Vincennes

\section{Édition imprimée}

Date de publication : 30 juin 2014

Pagination : 7-24

ISBN : 978-2-84292-405-8

ISSN : 0751-2708

\section{Référence électronique}

Christopher Lucken et Ludivine Jaquiery, «Harmonie et disharmonie », Médiévales [En ligne], 66 | printemps 2014, mis en ligne le 05 juillet 2016, consulté le 24 avril 2022. URL : http://

journals.openedition.org/medievales/7181; DOI : https://doi.org/10.4000/medievales.7181 
Christopher Lucken et Ludivine Jaquiery

\section{Harmonie et disharmonie}

«La consonance [consonantia] est l'accord [concordia], réduit à l'unité [in unum redacta], de sons dissemblables [dissimilium inter se vocum]», affirme Boèce dans son De institutione musica, traité de musique composé vers 510 qui synthétise les principes fondamentaux de la théorie pythagoricienne et platonicienne de la musique et qui, de l'époque carolingienne à la fin du Moyen Âge, servira de référence principale à toute réflexion relative à cette «discipline ${ }^{1}$. La consonance ne concerne donc pas tant les sons qui entretiennent une relation d'identité ou de similitude, que ceux qui se distinguent les uns des autres selon un rapport d'inégalité. Il n'y aurait pas besoin de consonance, en effet, s'il n'y avait au préalable une différence. La consonance désigne du même coup la résolution d'une disparité entre des sons distincts (comme entre toutes sortes d'éléments dissemblables), de sorte qu'ils s'unissent et composent un ensemble dont les différentes parties sont reliées de manière cohérente et forment ainsi, en quelque sorte, un seul et même son. C'est ce que souligne Boèce lorsqu'il dit que «les sons qui, faute de rapport d'inégalité, ne discordent pas (quae nulla inaequalitate discordant), ne réalisent aucune consonance du tout ${ }^{2} \gg$. Comme l'illustre de manière emblématique l'expression concordia discors, qui remonte à l'Antiquité, il n'y a pas d'harmonie sans disharmonie ${ }^{3}$.

1. BoÈce, Traité de la musique, I, III (trad. C. MeYer, Turnhout, 2004, p. 38-39). Sur la théorie musicale de Boèce et sa diffusion, voir en dernier lieu M. T. RimpLe, «The Enduring Legacy of Boethian Harmony», dans N. H. KaYlor, P. E. Phillips éd., A Companion to Boethius in the Middle Ages, Louvain, 2012, p. 447-478; sur la notion de consonance - et de dissonance - chez Platon, $c f$. C. LuCKEN, «Consonance-Dissonance. De Platon à Nietzsche», dans C. Lucken, J. Rigoli éd., Du Bruit à l'œuvre. Vers une esthétique du désordre, Genève, 2013, p. 191-241 (p. 197-213).

2. Ibid.

3. Sur la notion d'harmonie (au Moyen Âge et au-delà), $c f$. en particulier L. SPITZER, Classical and Christian Ideas of World Harmony, Baltimore, 1963, ouvrage tiré de deux études parues en 1944 et en 1945 dans la revue Traditio et qui a récemment fait l'objet d'une 


\section{Concordia discors}

C'est à ce célèbre syntagme qu'est consacrée l'étude d'Amy Heneveld qui ouvre le dossier thématique de ce numéro de Médiévales ${ }^{4}$. Cette dernière en retrace et en analyse un certain nombre d'emplois significatifs, de l'Antiquité au XIII ${ }^{\mathrm{e}}$ siècle, dus à différents auteurs qui l'appliquent non seulement à la musique, mais aussi à l'écriture, aux multiples textes de lois chargés de régir la vie des hommes ${ }^{5}$, aux relations de l'âme et du corps comme à celles entre les différents éléments du corps, aux diverses composantes de la société et au monde entier ${ }^{6}$. Si tout ensemble est constitué d'éléments distincts et potentiellement discordants, il est également gouverné par un principe de concorde qui en assure l'unité - principe qui peut également être identifié à l'amour ${ }^{7}$.

Toute dissonance finit-elle pour autant par former une consonance? La disharmonie est-elle toujours destinée à devenir harmonieuse? Le caractère déplaisant de nombreuses productions sonores semble y contredire. $\mathrm{Si}$ « une douce cantilène charme les jeunes enfants», affirme Boèce, «un air rude et sauvage [aliquid vero asperum atque inmite] interrompt le plaisir de l'audition ${ }^{8} »$. Le plaisir ou le déplaisir que suscite un ensemble de sons

excellente traduction française annotée: L'Harmonie du monde. Histoire d'une idée, trad. G. Firmin, Paris, 2012; voir aussi le catalogue de l'exposition organisée par la Cité de la musique: Moyen Âge entre ordre et désordre, Paris, 2004.

4. Les six études qui composent ce dossier thématique sont tirées d'une Journée d'études de l'Association des Jeunes Chercheurs Médiévistes de l'Université de Genève, organisée le 11 mai 2012 par Ludivine Jaquiery et Dora Kiss Muetzenberg. Nous remercions vivement le Département de langues et de littératures françaises et latines médiévales de l'Université de Genève pour son soutien financier à la publication de cet ensemble, et plus particulièrement, Genève pour son soutien financier à la publication de cet
pour le suivi scientifique, le Professeur Jean-Yves Tilliette.

pour le suivi scientifique, le Professeur Jean-Yves Tilliette.
5. Comme le souligne notamment le titre habituellement donné au Moyen Âge au 5. Comme le souligne notamment le titre habituellement donné au Moyen Âge au
Décret de Gratien (XII siècle): Concordia discordantium canonum. Cf. S. KuTtNER, Harmony from Dissonance: An Interpretation of Mediaeval Canon Law, Latrobe (PA), 1960, repris dans History of Ideas and Doctrines of Canon Law in the Middle Ages, Londres, 1980; H. de LuBAC, «À propos de la formule diversi sed non adversi», dans Mélanges Lebreton, Recherches de science religieuse, 39-40 (1951-1952), t. II, p. 27-40.

6. Notamment à travers la théorie pythagoricienne de l'harmonie des sphères: parmi les nombreuses études sur le sujet, $c f$. P. Boyancé, «Les Muses et l'harmonie des sphères",

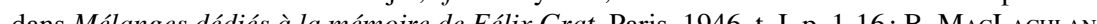
dans Melanges dedies à la sén "The Harmony of the Spheres: dulcis somus", dans R. W. WaLLACE, B. MAcLschlan éd. Harmonia mundi. Musice e filosofia nell Antichità, Rome, 1991, p. 7-20; M. ArmisenMarchetti, «L'harmonie des sphères chez Macrobe, Comm. II, 1-4», dans P. Defosse éd., Hommages à Carl Deroux, t. IV, Archéologie et Histoire de l'Art, Religion, Bruxelles, 2003, p. 268-282.

7. Cf. C. J. De Vogel, «Amor, quo caelum regitur», Vivarium, 1 (1963), p. 1-40; P. DRONKE, «L'amor che move il sole e l'altre stelle», Studi medievali, 6 (1965), p. 389-422.

8. Bö̀cE, Traité de la musique, I, I, p. 28-29. paraît à première vue susceptible de déterminer son caractère harmonieux ou disharmonieux ${ }^{9}$.

La consonance est la fusion [mixtura] d'un son aigu et d'un son grave parvenant aux oreilles de manière agréable et homogène [suaviter uniformiterque]. La dissonance en revanche est le choc rude et désagréable [aspera atque iniucunda] de deux sons entremêlés [permixtorum] venant frapper l'oreille. Tant que ces sons ne veulent pas se mélanger [misceri] les uns aux autres et que chacun, en quelque sorte, s'efforce d'atteindre l'oreille en demeurant intact, et que l'un s'oppose à l'autre, ils parviennent, en effet, l'un et l'autre aux sens de manière désagréable.

À la fois douce et uniforme, la consonance séduit les sens et s'avère source de plaisir; la dissonance, au contraire, âpre et agressive, ne peut que gêner l'audition et déplaire. Boèce refuse cependant de fonder son jugement sur de simples sensations. $\mathrm{Si}$ «le sens de l'ouïe reconnaît la consonance», c'est à la raison d'en apprécier le «poids» et la véritable nature ${ }^{10}$ :

Chaque fois que l'on tend deux cordes, l'une étant plus grave, qu'elles sont mises en vibration simultanément et qu'elles font entendre un son pour ains dire homogène et agréable [permixtum quodammodo et suavem sonum] les deux voix vont alors se fondre pour n'en former qu'une, comme si elles étaient jointes l'une à l'autre [duaeque voces in unum quasi coniunctae coalescunt]: on obtient alors ce que l'on appelle une consonance. En revanche, lorsqu'elles sont mises en vibration simultanément, que chacune semble aller son propre chemin et qu'elles ne se mélangent pas [nec persmiscent] pour offrir à l'oreille un son agréable et unique composé de deux autres [suavem atque unum ex duobus compositum sonum], il y a alors ce que l'on appelle une dissonance.

La raison et les sens semblent, cependant, parfaitement s'accorder: les sons agréables à l'oreille s'avèrent homogènes, tandis que ceux qui la blessent demeurent distincts. Si la consonance est le produit de sons qui,

9. «Consonantia est acuti soni gravisque mixtura suaviter uniformiterque auribus accidens. Dissonantia vero est duorum sonorum sibimet permixtorum ad aurem veniens aspera tque iniucunda percussio. Nam lom sibinet misceri nolunt et quodmmodo integer uterque atque inicunda percussio. Namn dam sibine nisceri nolunt et quodammodo integer uterque nititur pervenire, cumque alter alteri officit, ad sensum insuaviter uterque transmittitur.» (bid., I, vIII, p. 44-45.)

10. «Consonantiam vero licet aurium quoque sensus diiudicet, tamen ratio perpendit. Quotiens enim duo nervi uno graviore intenduntur simulque pulsi reddunt permixtun quodammodo et suavem sonum, duaeque voces in unum quasi coniunctae coalescunt; tunc fit ea, quae dicitur consonantia. Cum vero simul pulsis sibi quisque ire cupit nec permiscent ad aurem suavem atque unum ex duobus compositum sonum, tunc est, quae dicitur dissonantia.» (Ibid., I, XXVIII, p. 84-85.) 
bien que différents, se mélangent au point de constituer une parfaite unité, la dissonance est le résultat de sons qui ne parviennent pas à se mêler et à fusionner au sein d'une même composition. Les sons qui donnent lieu à une dissonance se refusent ou ne réussissent pas à s'attacher et à faire lien. Ils préfèrent rester seuls, séparés les uns des autres, chacun luttant contre les sons produits en même temps que lui, comme s'ils étaient ennemis, afin de conserver son inaliénable et inégalable intégrité, cherchant à se faire entendre sans entrer en résonance avec quelque autre que ce soit, ni subir son influence.

Aussi, bien que toute consonance soit nécessairement fondée sur des sons différents, tous les sons ne sont pas liés les uns aux autres selon une relation telle qu'ils sont capables de donner forme à une semblable association. Encore faut-il, en effet, qu'ils entretiennent entre eux un rapport particulier fondé sur des proportions numériques définies. Comme le souligne Macrobe dans son Commentaire au Songe de Scipion (ca 430), à propos du son «qu'engendre un choc quelconque de l'air» et qui peut produire «soit un effet sonore doux et musical [dulce [...] et musicam], soit une résonance incongrue et désagréable [ineptum et asperum]», «si le choc est réglé par une loi numérique précise, il en résulte une modulation structurée et harmonieuse [compositum sibique consentiens modulamen]; mais lorsque le bruit résulte d'un heurt désordonné et n'obéissant à aucune mesure [tumultaria et nullis modis gubernata collisio], c'est un fracas confus et informe [fragor turbidus et inconditus] qui blesse $1^{\prime}$ 'oreille ${ }^{11} \gg$. C'est ce qu'aurait compris Pythagore, en parvenant à dégager les règles numériques sur lesquelles reposent les principales consonances. D'après la légende que rapporte Boèce, ce dernier aurait découvert «la proportionnalité qui fonde la concorde des sons [haec sonorum concordia iungeretur]», après avoir entendu «des coups de marteaux dont les divers sons produisaient en quelque sorte un accord harmonieux [ex diversis sonis unam quodam modo concinentiam]» et en avoir étudié les caractéristiques ${ }^{12}$. Une telle proportionnalité est définie par les intervalles qui séparent un son d'un autre. Tous les intervalles ne se prêtent pas, cependant, à la consonance. En effet, comme le souligne Martianus Capella dans le Livre IX des Noces de Philologie et de Mercure (composé entre 410 et 429), consacré à la figure d'Harmonie, «les uns sont appelés $\lambda \sigma \gamma \iota \chi \alpha ́$ [rationels], et les autres, $\alpha \dot{\lambda} \mathrm{o} \gamma \alpha$ [irrationnels]: sont rationnels ceux dont nous pouvons faire reposer la consonance sur une proportion; sont irrationnels ceux qui ne sont pas fondés sur un rapport numérique [ratio]; de même, les uns sont consonants,

11. Macrobe, Commentaire au Songe de Scipion, II, 1.5-6 (éd. et trad. M. ArmisenMarchetTi, Paris, t. II, 2003, p. 3).

12. Bò̇ce, Traité de la musique, I, x, p. 48-49. Cf. en particulier B. Van Mymeersch, «La philosophie pythagoricienne du nombre et la musique», Revue belge de musicologie, 51 (1997), p. 5-16. les autres dissonants [alia convenientia, alia discrepantia $][\ldots]^{13} »$. Mais, tandis que «le nombre des intervalles dissonants est excessivement grand, en revanche les intervalles consonants sont au nombre de six dans chaque trope »: soit la quarte, la quinte et l'octave, auxquels s'ajoutent ces trois mêmes intervalles augmentés chacun d'une octave ${ }^{14}$. Ce sont là, pour la tradition pythagoricienne, les seuls proportions ou intervalles rationnels susceptibles de produire une musique harmonieuse ${ }^{15}$. Aussi, selon Boèce pour qu'à partir d'une corde frappant l'air à plusieurs reprises et produisant de nombreux sons un seul son parvienne aux oreilles, il faut que «les vibrations des sons graves» soient «commensurables avec les vibrations des sons aigus, comme dans les rapports dont il a été question plus haut». Seule la présence d'une telle «commensuration [commensuratio]», c'est-àdire d'intervalles fondés sur des rapports numériques impliquant des unités de mesure communes, permet à des sons différents de réaliser «une fusion» et d'être réunis «en une seule consonance ${ }^{16} »$. C'est ainsi que la discordia peut être englobée dans une concordia qui l'empêchera de n'être jamais que disharmonie, désaccord et destruction.

Cette conception pythagoricienne de la consonance (et de la dissonance) est résumée par Boèce au début du Livre IV de son $D e$ institutione musica ${ }^{17}$.

Tout son semble composé, comme s'il était formé de certaines parties. Toute conjonction de parties [partiuum coniunctio] est assurée par un certain nombre de rapports [proportionibus]. La conjonction de sons, par conséquent, est formée de rapports. Or les rapports sont généralement évalués à l'aide de nombres. On trouve un rapport simple entre les nombres dans les multiples, les superpartiels ou les superpatients. Or les sons consonants ou dissonants sont perceptibles selon des rapports multiples ou superpartiels.

13. Martianus Capella, Les Noces de Philologie et Mercure, t. IX, Livre IX, L'harmonie (éd. et trad. J.-B. Guillaumin, Paris, 2011, § 949, p. 46-47)

14. Ibid., § 950, p. 47-48.

15. Comme le souligne Boèce au terme de son De arithmetica (Institution arithmétique II, 54.1 et 8, éd. et trad. J.-Y. Gulllaumin, Paris, 2002, p. 174 et 177), la quarte (diatessaron), la quinte (diapente) et l'octave (diapason) constituent l'ensemble des «accords musicaux » (omnes musicas consonantias), soit les trois intervalles permettant une «harmonie suprême et parfaite» (maxima perfectaque armonia).

16. Bö̀ce, Traité de la musique, I, xxxxi, p. 88-89.

17. «[...] omnis sonus quasi ex quibusdam partibus compositus esse videatur. Omnis autem partium coniunctio proportionibus constituta est. Sonorum igitur coniunctio proportionibus constituta est. Proportiones autem principaliter in numeris considerantur. Proportio vero simplex numerorum vel in multiplicibus vel in superparticularibus vel in superpartientibus invenitur. Secundum multiplices vero proportiones vel superparticulares consonae vel dissonae voces exaudiuntur. Consonae quidem sunt, quae simul pulsae suavem permixtumque inter se coniungunt sonum. Dissonae vero, quae simul pulsae non reddunt suavem neque permixtum sonum.» (Ibid., IV, I, p. 224-225.) 
Les sons consonants sont ceux qui, émis en même temps, se mêlent pour former une sonorité agréable et homogène [suavem permixtumque inter se coniungunt sonum]. Les sons dissonants sont ceux qui, émis en même temps, ne produisent pas une sonorité agréable ou homogène.

Conformément à la découverte de Pythagore, les consonances correspondent à une série de proportions numériques à la fois simples et mesurables. Si elles sont agréables, alors que les dissonances sont généralement désagréables, il ne suffit donc pas d'y trouver un certain plaisir: les sensations peuvent tromper ou être source de confusion, alors que la raison, procédant de manière rigoureuse à l'aide du calcul, ne saurait se laisser induire en erreur par des impressions. Seuls les sons régis entre eux par des rapports numériques déterminés sont consonants ${ }^{18}$ :

Bien que l'oreille semble percevoir quelque chose de consonant lorsque l'on rapporte à un son un petit son distant de deux tons et d'un demi-ton entier, il est démontré que cela n'est pas consonant par nature. Mais puisqu'aucun sens ne parvient à saisir les choses très petites, aussi le sens de l'ouïe ne parvient-il à distinguer la différence qui se présente au-delà du consonant. Elle pourra cependant être perçue si cette particule grossit par la répétition incessante des mêmes erreurs. Car ce que l'on a du mal à percevoir dans la chose la plus petite se distingue parfaitement une fois assemblé et réuni, et que cela commence à prendre une certaine ampleur.

Si une consonance implique par définition des sons entretenant un rapport d'inégalité, elle doit également répondre à une forme d'égalité, soit à un rapport numérique fondé sur des mesures communes. Elle ne saurait donc relier entre eux des éléments qui seraient simplement différents et dont la varietas ou la diversité se suffirait à elle-même. Les sons dont les relations demeurent étrangères à de telles proportions, aussi infime ou imperceptible que soit l'écart qui les caractérise, ne peuvent que s'avérer dissonants. Il n'y a pas, de ce point de vue, d'autre harmonie que celle gouvernée par la raison des nombres. Tout ce qui échappe à un tel principe de régulation ne peut qu'être - ou rester - marqué par la disharmonie.

Ces réflexions ne sauraient être réduites à la seule musique (au sens relativement réduit que l'on donne aujourd'hui à ce terme). «Aucune discipline ne peut $[. .$.$] être parfaite sans la musique», affirme par exemple$

18. «Quodsi videtur auribus consonum aliquid canere, cum cuilibet voci duos tonos ac semitonium integrum distans vocula comparetur, id non esse consonum natura monstratur; sed quoniam sensus omnis, quae minima sunt, comprehendere nequeat, idcirco hanc differentiam, quae ultra consonum procedit, sensum aurium non posse distinguere, fore autem ut decrescat errores. Nam quod in minimo haud sane cernitur compositum coniunctumque, cum iam magnum esse coeperit, pervidetur.» (Ibid., III, I, p. 170-173.)
Isidore de Séville dans son De musica, soit la section consacrée à cet ar des Étymologies (composées dans le premier quart du $\mathrm{VII}^{\mathrm{e}}$ siècle); car poursuit-il, «rien n'existe sans elle. La composition du monde lui-même, dit-on, obéit à une sorte d'harmonie de sons [ipse mundus quadam armonia sonorum fertur esse compositus] et la révolution du ciel lui-même est soumise à l'harmonie musicale [sub armoniae modulatione revolvi $]^{19}{ }^{\prime}$. Fondée sur le cours des sphères célestes reliées les unes aux autres selon des proportions et une cohésion exemplaires, la musique désigne avant tout le principe harmonique qui doit gouverner le monde tout entier - de la nature aux sociétés humaines ${ }^{20}$. «Musique du monde » créé par le Démiurge (selon le Timée de Platon), elle s'applique aussi bien aux astres qu'aux quatre éléments dont tout corps serait composé et aux quatre saisons; "musique de l'homme», elle permet aux différentes parties du corps et de l'âme dont ce dernier est constitué de rester unies et de se maintenir en équilibre «selon un ordre soigneusement établi »; «musique instrumentale», elle s'applique à la voix humaine comme aux instruments de musique ${ }^{21}$. Nature, dans le Roman de la Rose de Jean de Meun (XIII ${ }^{\mathrm{e}}$ siècle), ne manquera pas de souligner à son tour le rôle déterminant des «cors du ciel», «Qui tornoien en leur esperes, / Si com l'establi Dieus li peres», au moment d'exposer à l'amant les principes harmoniques qui régissent toute chose relevant de son pouvoir de création ${ }^{22}$.

La font entr'euls leur armonies,

Qui sont causes de melodies

Et des diversetez de ton

Que par acordances metons

En toutes manieres de chant :

N'est riens qui par celes ne chant.

19. Isidore de SÉviLle, Étymologies, Livre III, Les mathématiques, 16.1 (éd. et trad. G. Gasparotto et J.-Y. GullLaumin, Paris, 2009, p. 58-59).

20. Comme le souligne par exemple Cicéron dans le De republica (II, 42), cité par sain Augustin dans son De civitate Dei (II, 21) : «De même que dans le jeu des lyres et des flûtes, dans l'expression du chant et des voix, il faut garder un certain accord entre les divers sons émis, sans quoi ils se rendent insupportables aux oreilles délicates par leur confusion et leur discordance, tandis que, grâce à l'équilibre des voix différentes, le concert devient harmonieux et concordant; de même, par les relations bien réglées entre les ordres supérieurs, moyens et infieurs, come paries parties les plus differtes [moderata ratione civitatem consensu dissimillimorum concinere] Ce que le musiciens appellent harmonie dans le chant, s'appelle concorde dans la cité. (Saint Augustin, Le Cité de Dieu, éd. B. Dombart, A. Kalb, trad. G. Combès, Paris, 1959, t. I, p. 370-371.) Cf. P. Morpurgo, L'Armonia della natura e l'ordine dei governi (secoli XII-XIV), Florence, 2000.

21. BOĖCE, Traité de la musique, I, II, p. 32-35.

22. Guillaume de Lorris et Jean de Meun, Le Roman de la Rose, v. 16949-16978 (éd. et trad. A. Strubel, Paris, 1992, p. 980-981). 
Et muent par leur influances

Les accidenz et les sustances

Des choses qui sont souz la lune.

Par leur diversite commune

S'espaissent li cler element;

Clers font li espes ensement.

Et froit et chaut et sec et moiste

Tout aussi comme en une boiste

Font il en chascun cors venir

Pour leur parz ensamble tenir.

Tout soient il contrariant,

Les vont ensamble liant

Si font pais de .IIII. anemis

Quant si les ont ensamble mis

Par atrempance couvenable

A complession raisonnable,

Pour former en la meilleur forme

Toutes les choses que je forme.

C'est là qu'ils produisent entre eux leurs harmonies, causes des mélodies et des diverses sortes de tons que nous mettons, par les accords, en toutes formes de chants : il n'est rien qui par elles ne chante. De plus, ils modifient par leurs influences les accidents et les substances des choses qui existent sous la lune. Dans leur diversité commune, ils rendent opaques les éléments transparents et de la même façon ils rendent clairs les opaques. Et le froid, le chaud, le sec et l'humide, ils le font venir dans chaque corps, comme dans une boîte, pour qu'ensemble ils tiennent leur rôle. Bien qu'ils soient opposés, ils les lient entre eux en permanence, et font la paix entre quatre ennemis quand ils sont réunis selon un mélange convenablement proportionné et d'après un assemblage dicté par la raison pour donner la meilleure forme à toutes les choses que je forme.

Du fait que «l'âme du monde a été formée par une concorde musicale [musica convenentia fuisse coniunctam]» et «en vertu de ce qui est rassemblé en nous et harmonieusement agencé [iunctum convenienterque coaptatum]», comme l'affirme Boèce, lorsque «nous recevons ce qui dans les sons est justement et harmonieusement assemblé [apte convenienterque coniunctum], et que nous en sommes charmés, nous comprenons alors que nous-mêmes sommes constitués à sa ressemblance [eadem similitudine compactos]. La ressemblance, en effet, est l'amie [amica est enim similitudo], la dissemblance haïssable et néfaste [dissimilitudo odiosa atque contraria $]^{23} \gg$. Le plaisir que l'homme éprouve lorsqu'il entend des sons qui forment une agréable consonance tiendrait au fait qu'il subit ou qu'il perçoit, fût-ce inconsciemment, une harmonie similaire à celle qui préside à sa propre constitution. Non seulement la musique sonore est appelée à témoigner de l'harmonie qui ordonne l'univers, mais elle est aussi susceptible d'exercer une certaine influence sur les sentiments ou le comportement de chaque individu: «les rythmes et les modes descendent jusqu'à l'âme», souligne Boèce, de sorte qu'ils «affectent et façonnent l'esprit selon leur manière d'être ${ }^{24} \gg$. Ils permettent donc de rétablir l'harmonie que l'homme aurait perdue. Ainsi, par exemple, Pythagore est-il parvenu, à l'aide d'un mode adapté, à adoucir et à tempérer (temperare) l'âme d'un jeune homme excité et rendu furieux par le «mode phrygien » qu'il avait entendu précédemment, «jusqu'à ramener son esprit à un état totalement pacifié ${ }^{25}$ ».

\section{Ordre et beauté de la création divine}

La conception pythagoricienne de la consonance et de l'harmonie musicales sera reprise et prolongée dans une grande mesure par les Pères de l'Église ${ }^{26}$. Dieu, «éternel et suprême principe des harmonies, de la similitude, de l'égalité et de l'ordre [principatu numerorum et similitudinis et aequalitatis et ordinis]», comme l'écrit saint Augustin dans son De musica $(\mathrm{ca} 389)^{27}$, se substitue bien sûr à l'Âme du monde. «Divine Providence qui a créé et qui gouverne toutes choses», c'est Lui qui «fait que l'âme pécheresse et misérable, elle aussi, est réglée par des harmonies », en produisant «jusque dans les bas-fonds de la corruption charnelle; et ces harmonies certes peuvent être de moins en moins belles, mais elles ne peuvent manquer tout à fait de beauté [pulchritudine] ${ }^{28} \gg$. Quels que soient la déchéance et l'éloignement de Dieu qui la caractérisent, toute âme conserverait en elle la trace de cette harmonie qui fonde ici la beauté. Or, précise Augustin, «l'harmonie [numerus] commence par l'unité, elle tire sa beauté de l'égalité et de la symétrie [aequalitate ac similitudine], et l'ordre en est l'union [et ordine copulatur]». Aussi, étant donné que «tout ce qui

23. Bо亡̈с, Traité de la musique, I, I, p. 22-23.

24. Ibid.
25. Ibid., p. 26-27.

26. Cf. T. Gérold, Les Pères de l'Église et la musique, Strasbourg, 1931; J. McKinnon, Music in Early Christian Literature, Cambridge, 1987.

27. Saint Augustin, La Musique, VI, xviI, 58 (éd. et trad. G. Finaert et F.-J. Thonnard, Paris, 1947, p. 474-475)

28. Ibid., VI, XVII, 56, p. 470-71. Sur la notion de beauté au Moyen Âge, $c f$. E. DE BRUYNE, Études d'esthétique médiévale, Bruges, 1946 (3 vol.); U. Eco, Art et beauté dans l'esthétique médiévale [1987], trad. M. Javion, Paris, 2002. 
est, dans la mesure où il est, a été créé et façonné par un Unique principe au moyen d'une beauté qui est égale et semblable aux richesses de sa bonté », «aucune nature ne manque, pour être ce qu'elle est, de tendre vers l'unité, de s'efforcer, autant qu'elle peut, de rester semblable à elle-même, de garder son ordre propre, soit dans le temps, soit dans le lieu, soit en assurant sa conservation par un bon équilibre corporel $^{29}{ }^{2}$. C'est parce que l'homme désire l'unité, l'égalité et la similitude qui fondent l'harmonie, qu'il aime normalement les «belles choses», plutôt que les «choses laides» ou difformes (deformia) que semblent apprécier ceux que «les Grecs appellent vulgairement saprophiles», car personne, selon Augustin, «n'aime les objets dont la laideur [foeditate] blesse les sens ${ }^{30} »$. Or «ces choses belles plaisent par leur harmonie dans laquelle [...] nous recherchons l'égalité», tandis que «nous nous détournons [...] d'un éclat excessif et nous refusons de considérer ce qui est trop obscur; et de même, dans les sons, nous fuyons ceux qui sont excessifs et nous n'aimons pas ceux qui se réduisent à un bourdonnement ${ }^{31} \gg$ :

Ainsi donc, lorsque, dans ces objets, nous cherchons ce qui nous convient selon la mesure de notre nature et repoussons ce qui ne nous convient pas, sachant du reste qu'ils conviendraient à d'autres vivants, n'est-ce pas encore une certaine loi d'égalité qui nous plaît, quand nous reconnaissons que là aussi, par des moyens plus cachés, le semblable est mis en rapport avec le semblable? On peut observer ce fait dans les parfums, dans les saveurs et dans le toucher; il serait long d'en poursuivre l'exposé en détail, mais très facile de l'expérimenter. Car il n'y en a aucun parmi ces objets sensibles qui ne nous plaisent point par leur égalité, ou par leur similitude. Là où réside l'égalité et la similitude, là est le principe de l'harmonie et du nombre, car rien n'a autant d'égalité et de similitude que l'un comparé à l'un.

La beauté étant fondée sur l'harmonie du nombre, le plaisir que suscitent les choses sensibles serait dû à la similitude que l'homme y perçoit entre elles et lui. Mais si les «nombres sonores» sont «la cause de ces nombres qui sont reçus dans l'oreille quand nous entendons», selon Augustin, il faut leur préférer ceux qui sont produits dans la mémoire ${ }^{32}$. L'âme ne reçoit pas

29. Ibid. (nous avons interverti ici les deux moments de l'argumentation d'Augustin).

30. Ibid., VI, XIII, 38, p. 440-443 (saprophile signifie mot à mot «qui aime la laideur»), D'après Platon, «le défaut de grâce dans l'apparence, le manque de rythme et d'harmonie s'apparentent à la laideur du langage et du caractère» (PLATON, La République, III, 401a, trad. G. Leroux, Paris, 2002, p. 188). Sur la laideur du point de vue esthétique, $c f$. K. RosenKranz, Esthétique du laid [1853], trad. G. Raulet et S. Muller, Paris, 2004; U. Eco éd, Histoire de la laideur [2007], trad. M. BOUZAHER, Paris, 2007.

31. Ibid., p. 442-445

32. Ibid, VI, Iv, 7, p. 373-374. l'harmonie des corps; c'est elle qui les imprime plutôt «aux corps après les avoir reçues du Dieu souverain». Aussi l'âme devient-elle meilleure «en se dépouillant [des nombres ou des harmonies] qu'elle reçoit des corps, lorsqu'elle se détourne des sens charnels et se réforme selon les harmonie divines de la Sagesse ${ }^{33}$ ». Ce n'est donc pas la musique elle-même ou toute autre forme d'art et de manifestation sensible qui, pour Augustin, permet à l'homme de retrouver l'équilibre et la similitude qui doivent régir son être, mais l'harmonie avec laquelle Dieu façonne toute chose. La seule musique à même de pénétrer l'âme humaine sans risquer d'être pervertie ou désaccordée par les sensations serait alors celle des Anges, soit ces «harmonies rationnelles et intelligibles des âmes saintes et bienheureuses " qui, «accueillant sans aucun intermédiaire la loi même de Dieu [...], transmettent cette loi jusqu'à l'ordre établi sur terre et dans les enfers ${ }^{34} »$. «Rendues rauques par les soucis de la chair, les voix de la louange humaine ne suffisent pas à chanter la majesté divine», affirme à son tour un poème latin $\mathrm{du} \mathrm{XII}^{\mathrm{e}}$ siècle; c'est pourquoi cette dernière «a ordonné à la milice angélique de lui chanter aux cieux une sainte symphonie et de faire en sorte d'imposer, par son mouvement, une concorde harmonieuse aux diverses discordes du monde ${ }^{35} \gg$.

La musique vocale et instrumentale n'a pas été éliminée pour autant de l'Église chrétienne. Elle ne saurait toutefois se détacher de l'harmonie divine et c'est elle qu'il faut transmettre avant tout. Plusieurs figures emblématiques servent d'ailleurs à le rappeler. Ce sont deux d'entre elles qu'analyse ici Welleda Muller à partir des images qu'en offrent les manuscrits et les stalles gothiques répertoriés en France. La première est David, auteur emblématique des Psaumes. Connu pour avoir arraché «Saül

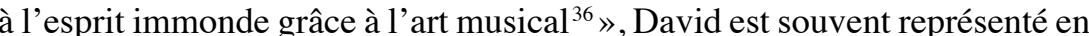
train d'accorder sa harpe. Il illustre ainsi symboliquement le rôle qui lui est attribué : celui de rendre au monde l'harmonie dont l'humanité aurait perdu la clé. La seconde figure est celle du chœur céleste des Anges musiciens, qui remplace en quelque sorte, pour la tradition chrétienne, l'harmonie des sphères issue de la pensée platonicienne. Comme l'affirme saint Augustin dans son De musica, les Anges sont chargés de communiquer aux hommes l'harmonie du Créateur dont ils sont les messagers en même temps qu'ils célèbrent sa gloire éternelle. Les images consacrées à ces deux

33. Ibid., p. 376-377.

34. SaINT Augustin, La Musique, VI, xVII, 58, p. 476-477.

35. «Voces laudis humane / curis carneis rauce / non divine maiestati / cantu sufficiunt, / Que angelicam / sibi militiam / in excelsis psallere / sanctam iussit / simphoniam / Nec non variam / mundi discordiam / se movendo concordem / dare fecit / armoniam.» (The Cambridge Songs (Carmina Cantabrigiensia), 3, 1-2, éd. et trad. anglaise J. M. ZıoLKOWSKI, New York/Londres, 1994, p. 4.)

36. Isidore de SÉville, Étymologies, III, 16.3, p. 58. 
figures ne peuvent faire entendre à proprement parler leur musique: elles n'en transmettent pas moins l'exigence d'une harmonie céleste qu'aucun instrument ni aucune voix ne saurait véritablement traduire de manière sonore.

C'est à une autre pratique «musicale» que s'intéresse Philip Knäble dans l'étude qu'il consacre à une danse paraliturgique pratiquée jusqu'au XVI ${ }^{\mathrm{e}}$ siècle par les membres du clergé d'Auxerre. Cette dernière a pour particularité d'avoir comme accessoire un ballon de grande taille. Plutôt que s'apparenter à un divertissement sportif ou à une fête courtoise, comme la célèbre carole du Jardin de Déduit dans le Roman de la Rose de Guillaume de Lorris, susceptible d'entraîner dans son cours délicieux ceux qui cèdent à son charme comme à leurs propres passions, cette danse semble au contraire représenter la danse des astres. En mimant le mouvement des sphères célestes, le clergé auxerrois célèbrerait le retour de l'harmonie, soit une victoire de l'amour et de la concorde sur les forces diaboliques de la discorde et de la division, d'autant qu'il effectue une telle chorégraphie à l'occasion de la fête de Pâques.

\section{L'harmonie à l'épreuve de la dissemblance}

«La ressemblance [...] est l'amie, la dissemblance haïssable et néfaste», affirme Boèce, qui justifie ainsi le fait que l'homme aime l'harmonie musicale et y trouve du plaisir, l'invitant du même coup à se rendre compte qu'il est composé à sa ressemblance. De façon analogue, la ressemblance ou la similitude (similitudo) est, pour saint Augustin, l'un des principes fondamentaux sur lesquels reposent l'harmonie et le nombre, l'attraction que suscite sa beauté incitant l'homme à se rapprocher de Celui qui en est la source et à l'image et à la ressemblance duquel il a lui-même été créé (d'après la Genèse, I, 26). En revanche, l'âme qui se disperse et perd tout équilibre ne fait que «rendre son Dieu lointain ${ }^{37} »$; aussi ne peut-elle que se retrouver séparée de Lui dans la «région de la dissemblance [regio dissimilitudinis]», un lieu sans limite, étranger à toute identité comme à toute forme définie par la ressemblance, où l'homme ne saurait éviter de s'égarer dans la confusion et la démesure ${ }^{38}$. Plutôt que de s'apparenter à Dieu, l'âme devient en quelque sorte semblable aux bêtes privées de raison,

37. SAint Augustin, La Musique, VI, xIII, 40, p. 446-447.

38. Saint Augustin, Les Confessions, VII, XI, 16 (éd. M. Skutella et trad. E. Tréhorel et G. Bouissou, Paris, 1962, t. I, p. 616-617). Sur la regio dissimilitudinis, expression d'origine platonicienne (Politique, 273d), cf. F. ChÂTtLlon, «Regio dissimilitudinis», dans Mélanges F. Podechard, Lyon, 1945, p. 85-102; É. GiLson, «Regio disimilitudinis de Platon à saint Bernard de Clairvaux», Mediaeval Studies, 9 (1947), p. 108-130; P. Courcelle, «Tradition néo-platonicienne et traditions chrétiennes de la "Région de dissemblance" (Platon, Politique, 273d)», Archives d'Histoire Doctrinale et Littéraire du Moyen Âge, 24 (1957), p. 5-33. comme l'affirme par exemple Guillaume de Saint-Thierry dans son De natura corporis et animae (ca 1138-1145) ${ }^{39}$.

Créée pour posséder l'image et la vision de Dieu, au moment où elle aurait dû commencer à goûter Dieu, [l'âme] commence à en perdre le goût. Se retirant «de la face de Dieu» comme Caïn, elle habite la région de la dissemblance, la terre de Naïm, c'est-à-dire de la commotion. Bannie de la vertu, elle se soumet aux vices, devenue étrangère à la paix des fils de Dieu, elle est agitée de troubles intérieurs, elle dévoie ses talents naturels en malice et en ruse, elle devient perverse, elle se plonge scandaleusement dans les attraits de la sensualité animale et s'adonne à la luxure. Elle devient «comme le cheval et le mulet qui n'ont aucune intelligence», habile seulement à faire le mal, mais ne sachant pas bien faire. Nul souci d'elle-même, aucun souvenir de Dieu.

L'homme apparaît sommé ici de choisir entre la ressemblance et la dissemblance comme entre l'harmonie et la disharmonie. Ce sont un tel objectif et une telle opposition que représente la Crucifixion attribuée au Maître de la Croix des Piani d'Invrea conservée au musée des Beaux-Arts de Tours ( ca 1330), commentée par Amélie Bernazzani dans son étude. Dans ce tableau, le bon larron offre des traits communs avec le Christ, tandis que le mauvais larron s'en distingue. De même, les spectateurs qui entourent le Christ présentent des caractères qui les éloignent de ce dernier ou, au contraire, qui l'en rapprochent, selon qu'ils appartiennent à ses ennemis ou à ses fidèles. L'image possède du même coup une portée didactique: elle incite ceux qui la contemplent à rejeter les personnages qui incarnent la laideur pour se conformer au Christ et à ceux dont la beauté ressemble à ce dernier et témoigne du fait qu'ils seront sauvés.

C'est, dans un tout autre contexte, une opposition analogue qu'analyse Ludivine Jaquiery dans son étude consacrée à deux personnages importants du roman de Lancelot en prose, l'un bon, Lancelot, l'autre mauvais, Claudas. Conformément à l'idée fortement répandue dans l'Antiquité et au Moyen Âge que le corps reflète l'âme, on s'attend à ce que le personnage moralement beau soit harmonieux dans ses proportions et ses dispositions corporelles, au contraire du personnage méchant. Pourtant, alors que le corps de Claudas est parfait, l'exemple de Lancelot à la trop large poitrine tend vers une valorisation de la démesure, en lien avec la force de l'amour qu'il porte à Guenièvre: aussi excessif que soit cet amour,

39. Gulllaume de Saint-Thierry, De la nature du corps et de l'âme, II, 118 (éd. et trad. M. Lemoine, Paris, 1988, p. 210-211); sur ce passage et l'origine néo-platonicienne de l'analogie avec les animaux, $c f$. P. CourCelle, Recherches sur les Confessions de saint Augustin, Paris, 1968, Appendice VII, «La "région de dissemblance" dans la tradition néoplatonisante», p. 405-440 (p. 431-433). 
il est un principe unificateur chez Lancelot, et c'est justement grâce à lui que le jeune homme dépasse les éléments contraires mêlés en lui, tandis que Claudas reste profondément duel et disharmonieux.

Comme nous l'avons déjà noté, l'harmonie concerne non seulement les instruments de musique et la structure du monde, mais aussi l'homme, véritable microcosme créé à la mesure du cosmos. Guillaume de SaintThierry le souligne après d'autres: «Les éléments opèrent dans le monde de la même façon que les quatre humeurs opèrent dans ce monde en réduction qu'est l'homme, ce microcosme dont j'ai parlé plus haut: ils s'accordent à partir de la diversité qui est entre eux [ex sua sibi diversitate concordantia], et, grâce à cette diversité concordante [per concordem diversitatem], rendent plus belle l'unité qui existe dans leur bon ordre ${ }^{40}$.» Une telle harmonie caractérise également l'apparence extérieure de l'homme. Guillaume de Saint-Thierry note à ce propos que son corps, «considéré dans le sens de la longueur», est caractérisé par «l'unité moyenne» de ses membres; qu'en largeur, on «retrouve une belle parité des membres»; et que «le corps tout entier est composé en poids, en mesure, en nombre» (comme le dit un célèbre verset de la Sagesse, XI, 21, habituellement convoqué pour souligner l'harmonie de la Création divine $)^{41}$. En ce qui concerne la mesure de l'homme, Guillaume de Saint-Thierry s'inspire de Vitruve (20 après J.-C.), qui affirme dans son traité De architectura que les différents membres de l'homme ont été ordonnés par la nature et que leur mesure correspond à une série de proportions bien définies (III, I, 3), conception encore connue aujourd'hui grâce au célèbre dessin qu'en a tiré Léonard de Vinci, intitulé habituellement «L'homme de Vitruve» $(\text { ca 1492 })^{42}$ :

Selon les physiciens, un homme étant couché sur le dos, mains et membres étendus, si l'on place un compas au centre de son nombril et qu'on en fasse faire à celui-ci un tour complet, on trouvera égalité et équilibre dans toutes les parties de cet homme, sans que le tracé de cette mesure connaisse la moindre altération.

40. Ibid., I, 11, p. 80-83. Cf. J. PIGEAUd, «Homo quadratus. Variations sur la beauté et la santé dans la médecine antique», Gesnerus, 42 (1985), p. 337-52; P. Morpurgo, L'Armonia degli «elementi». Fuoco, Aria, Acqua e Terra nei dibattiti salernitani, Salerne, 1993.

41. Ibid., I, 48, p. 122-125.

42. «Dicunt enim physici quia, si homo supinus extensis manibus et membris iaceat, si circinum in centro umbilici locatum undique circumvoluatur, inoffenso mensurae cursu in omnibus partibus suis, par sibi et aequalis inveniatur» (Ibid., I, 49, p. 124-125). Cf. F. ZöLlner, Vitruvs Proportionsfigur : Quellenkritische Studien zur Kunstliteratur im 15. und 16. Jahrhundert, Worms, 1987.
Si, à l'exemple de la Terre, l'homme semble correspondre au dessin tracé par le compas de Dieu ${ }^{43}$, il n'en est pas de même des monstres. Selon Varron, en effet, ces derniers sont nés contre nature. Isidore de Séville estime cependant, après avoir cité Varron dans ses Étymologies, que la nature de toute chose correspond à la volonté de Dieu, tout en concédant que les monstres sont contraires à ce que l'on connaît de la nature (XI, III, 1-2). Isidore de Séville s'appuie vraisemblablement sur saint Augustin, qui affirme par exemple, dans son De ordine, que rien ne saurait être étranger à l'ordre gouverné par la providence divine. Lorsque l'homme, «avec un esprit étroit», considère en elle même quelque chose d'une grande laideur, note Augustin, il s'en détourne et est repoussé par elle (magna repercussus foeditate aversetur). "Mais si, élevant les yeux de l'esprit et les portant de divers côtés, il parcourt tout en même temps du regard, il ne trouvera rien qui ne soit ordonné, toujours distribué et disposé pour ainsi dire à sa place $^{44}$.» Il en est ainsi, par exemple, des bourreaux, des courtisanes, de certains membres du corps humain comme des solécismes et barbarismes qu'affectionnent les poètes: «Retire-les des poèmes: nous regretterons des assaisonnements très délicieux. Amasses-en beaucoup en un seul endroit l'ensemble âcre, puant, rance me dégoûtera. [...] Ainsi l'ordre qui gouverne et règle ces choses n'admettra ni qu'elles soient excessives chez elles ni étrangères en n'importe quel endroit ${ }^{45}$.» Malgré leur caractère discordant et le risque de provoquer la nausée si elles dominent le reste, de telles «monstruosités » doivent pouvoir entrer en consonance avec l'ensemble et s'intégrer dans son ordre. Augustin rejoint en quelque sorte Aristote qui, dans Les Parties des animaux, affirme qu'il faut étudier ces derniers «sans répugnance, parce que chez absolument tous il y a quelque chose de naturel, c'est-à-dire de beau. Car dans les œuvres de la nature, ce n'est pas le hasard qui est présent, mais le "en vue de quelque chose", et on le trouve avant tout là. Et la fin en vue de laquelle un être a été constitué ou est venu à l'être tient la place du beau ${ }^{46} »$.

C'est à une des catégories de monstres identifiées par Isidore de Séville que s'intéresse Pascale Tiévant, celle des nains (nanus) (XI, III, 7). Elle étudie pour cela les enluminures de différents types d'œuvres en langue française, romanesques ou non, contenues dans un certain nombre de manuscrits des $\mathrm{XIV}^{\mathrm{e}}$ et $\mathrm{XV}^{\mathrm{e}}$ siècles en France. Bien qu'il soit le plus

43. Cf. J. B. Friedman, «The Architect's Compass in the Creation Miniatures of the Later Middle Ages», Traditio, 30 (1974), p. 419-429; F. BoEsPFLug, «Le Créateur au compas. Deus geometra dans l'art d'Occident», Micrologus, 19 (2011), p. 113-130.

44. Saint Augustin, De Ordine, II, 11 (éd. et trad. J. Doignon, Paris, 1997, p. 196-197; $c f$. aussi la note complémentaire).

45. Ibid., II, 12-13, p. 200-201.

46. Aristote, Les Parties des animaux, 645a 23-25 (trad. P. Pellegrin, Paris, 2011 p. 131) 
souvent considéré comme un être dépourvu de mesure, du fait d'une taille plus petite que celle qu'atteignent normalement les hommes et d'une tête disproportionnée par rapport au reste du corps, le nain n'est pas toujours représenté, dans les images de cette période, comme un personnage difforme; il peut même apparaître comme un être humain en miniature et se voir pourvu de proportions relativement harmonieuses.

Une telle tendance ne serait-elle pas une conséquence de la beauté qu'on attend d'une enluminure ? La qualité d'une peinture ne dépend pas de l'apparence agréable de ce qu'elle montre et la laideur d'un sujet n'implique pas nécessairement celle de sa représentation. Comme le note Aristote dans sa Poétique, l'homme peut aimer «contempler l'image exécutée avec la plus grande exactitude» d' «êtres dont l'original fait peine à la vue»: «par exemple les formes des animaux les plus vils et les cadavres ${ }^{47} \gg$. Le plaisir que suscite une image - comme toute autre forme d'imitation - rejaillit en quelque sorte sur ce qu'elle représente, au point de transformer ce qui est laid en quelque chose de beau.

Les défauts ou le caractère disharmonieux d'un sujet ne risquentils pas, cependant, de brouiller la notion même de beauté et d'affecter la forme harmonieuse à laquelle semble devoir se conformer une œuvre d'art? Socrate préconise, dans la République de Platon, de contraindre les poètes à «ne présenter dans leurs compositions poétiques que les images du caractère vertueux » et de «soumettre aussi à des règles les autres artisans », en leur interdisant notamment «de représenter dans leur production le caractère vicieux, l'intempérance, la servilité, l'absence de grâce, que ce soit dans les images des êtres vivants, dans l'architecture, ou dans tout autre genre de représentations artisanales». Il s'agit d'éviter ainsi que les gardiens de la cité idéale «soient élevés au milieu des images du vice» et qu'ils «finissent [...] par amasser dans leur âme un mal immense»; il s'agit du même coup de les disposer «insensiblement à la ressemblance, à l'amour et à l'harmonie avec la beauté de la raison ${ }^{48} »$. Aussi Socrate estime-t-il que «les plaintes funèbres et les lamentations ne conv[iennent] pas à nos compositions», qu'elles sont «inutiles, même pour les femmes qui doivent être décentes, pour ne rien dire des hommes », et qu'il faut donc les «éliminer ${ }^{49} »$. «L'homme sage» ne doit pas considérer «le fait de mourir comme une chose terrible», même si la personne concernée lui est très

47. ARIStote, La Poétique, 4, 1448b (trad. J. HARDy, Paris, 1932, p. 33).

48. Platon, La République, III, 401b-d, p. 188-189.

49. Ibid., 398de, p. 184 ( $c f$. aussi 387c-388a). On retrouve de semblables condamnations dans la tradition chrétienne. Par exemple, Clément d'Alexandrie demande dans les Stromates (VI, 90) de bannir «la musique qui est trop artificielle, qui brise les âmes et les entraîne à trop de sentiments divers, tantôt larmoyante, tantôt impudique et voluptueuse, tantôt provoquant une fureur bachique et l'insanité» (cité d'après T. GérolD, Les Pères de l'Église et la mиsique..., p. 89). proche; et «s'il doit affronter une situation malheureuse, il le supportera le plus sereinement du monde ${ }^{50} \gg$. La souffrance, comme tout ce qui contredit l'harmonie à laquelle l'homme doit soumettre son âme, ne saurait faire l'objet d'une représentation ou être exprimée dans une œuvre d'art. Ains que l'illustrent les anecdotes attribuées à Pythagore (ou à David), l'art ou la musique doivent servir au contraire à apaiser les hommes et à rétablir l'harmonie que leur douleur met à mal.

Comment manifester la tristesse que suscite la mort d'un compositeur sans porter atteinte à la beauté musicale dont il est la source et la figure emblématique? Tel est en quelque sorte le dilemme que rencontre Guillaume Crétin en écrivant sa Déploration sur le trépas de Jean Okeghem, poème qu'analyse Thibaut Radomme dans la dernière étude de cet ensemble. Si la disharmonie des cœurs paraît devoir être résorbée dans l'harmonie des chœurs, comment celle-ci pourrait-elle ne pas être affectée par la disharmonie qui motive l'existence même de ce planctus? D'autant plus que, la figure du musicien ayant désormais disparu, le poète se retrouve, pour ainsi dire, privé de musique et contraint d'employer un langage menacé du même coup de se réduire à de simples cris. Impossible, dans ces conditions, de réaliser l'œuvre qu'on attend de lui ! À moins de faire de la poésie, au contraire d'une musique ne pouvant que célébrer la joie, le moyen par lequel la disharmonie des sentiments et l'harmonie de la composition trouvent à s'accorder en quelque sorte sous la forme paradoxale d'une chantepleure, soit d'un chant qui pleure et qui devient ainsi une expression caractéristique de la concordia discors ${ }^{51}$

Si la disharmonie s'oppose à l'harmonie, elle semble également destinée à être résorbée par son contraire. Rien ne semble pouvoir échapper à la puissance unifiante du nombre telle que la conçoit l'esthétique médiévale, pas plus qu'à l'ordre fondé sur l'amour de Dieu. La concorde harmonieuse venue de la symphonie des chœurs célestes, précise le poème latin cité précédemment, «a protégé des morsures des loups ses agneaux lavés à la source, leur assurant une pieuse paix en raffermissant l'Empire romain ${ }^{52} \gg$. De même que les morsures des loups, la discorde et la disharmonie ne cessent pourtant de revenir mettre en cause la cohérence et la beauté de la Création divine. Tel est aussi bien souvent le destin des œuvres que réalisent les hommes. Quelle est donc la «cause discordante» qui rompt «les alliances du monde ?», demande Boèce dans la Consolation

50. Ibid., $387 \mathrm{de}$, p. 166

51. Cf. en particulier M. ZinK, «Un paradoxe courtois: le chant et la plainte», dans D. Maddox, S. Sturm-Maddox éd., Literary Aspects of Courtly Culture, Cambridge, 1994 p. 69-83.

52. «[...] Que imperium / confirmando Romanum / suos agnos / fonte lotos / a luporum morsibus / pia pace / custodivit» (The Cambridge Songs (Carmina Cantabrigiensia), 3, 3, p. 4). 
de Philosophie ${ }^{53}$. A défaut d'une réponse définitive, on peut toujours confier aux consonances musicales le pouvoir de vaincre le mal. Mais l'harmonie est sans cesse à recommencer.

Christopher LUCKEN - Université Paris 8, Département de littératures française et francophone; Université de Genève, Département de langues et littératures françaises et latines médiévales

Ludivine JAQUIERY - Université de Genève, Département de langues et littératures françaises et latines médiévales

53. «Quaenam discors foedera rerum / causa resolvit?» (Bò̀CE, La Consolation de Philosophie, VI, 3, v. 1-2, éd. C. Moreschini, trad. É. VAnPeTEGHem, Paris, 2005, p. 292-293). 